\title{
Transparent and flexible, nanostructured and mediatorless glucose/oxygen enzymatic fuel cells
}

\author{
Dmitry Pankratov a, b, Richard Sundberg ' , Javier Sotres ${ }^{\text {a }}$, Ivan Maximov ${ }^{\text {c }}$, \\ Mariusz Graczyk c, Dmitry B. Suyatin c, e, Elena González-Arribas a, Aleksey Lipkin d, \\ Lars Montelius ${ }^{c, e}$, Sergey Shleev ${ }^{a, b, ~ d, ~ * ~}$ \\ ${ }^{a}$ Biomedical Sciences, Health and Society, Malmö University, 20560 Malmö, Sweden \\ ${ }^{\mathrm{b}}$ A.N. Bach Institute of Biochemistry, 119071 Moscow, Russia \\ ' Division of Solid State Physics and the Nanometer Structure Consortium (nmC@LU), Lund University, 22100 Lund, Sweden \\ d Kurchatov NBIC Centre, National Research Centre "Kurchatov Institute", 123182 Moscow, Russia \\ e Neuronano Research Center, Medical Faculty, Lund University, 22100 Lund, Sweden
}

\section{H I G H L I G H T S}

- Transparent and flexible enzymatic fuel cells were fabricated and characterised.

- The glucose/oxygen utilising devices were nanostructured, membrane-less and mediator-free.

- Nanoimprint lithography was used for nanostructuring of polymer based electrodes.

- Nanostructured electrodes were metallised and biomodified.

- This type of biodevice could potentially be used in smart electronic contact lenses.

\section{A R T I C L E I N F O}

\section{Article history:}

Received 27 February 2015

Received in revised form

24 May 2015

Accepted 8 June 2015

Available online 28 June 2015

\section{Keywords:}

Enzymatic fuel cell

Flexible

Transparent

Nanostructured

Mediatorless

\begin{abstract}
A B S T R A C T
Here we detail transparent, flexible, nanostructured, membrane-less and mediator-free glucose/oxygen enzymatic fuel cells, which can be reproducibly fabricated with industrial scale throughput. The electrodes were built on a biocompatible flexible polymer, while nanoimprint lithography was used for their nanostructuring. The electrodes were covered with gold, their surfaces were visualised using scanning electron and atomic force microscopies, and they were also studied spectrophotometrically and electrochemically. The enzymatic fuel cells were fabricated following our previous reports on membrane-less and mediator-free biodevices in which cellobiose dehydrogenase and bilirubin oxidase were used as anodic and cathodic biocatalysts, respectively. The following average characteristics of transparent and flexible biodevices operating in glucose and chloride containing neutral buffers were registered: $0.63 \mathrm{~V}$ open-circuit voltage, and $0.6 \mu \mathrm{W} \mathrm{cm}{ }^{-2}$ maximal power density at a cell voltage of $0.35 \mathrm{~V}$. A transparent and flexible enzymatic fuel cell could still deliver at least $0.5 \mu \mathrm{W} \mathrm{cm}{ }^{-2}$ after $12 \mathrm{~h}$ of continuous operation. Thus, such biodevices can potentially be used as self-powered biosensors or electric power sources for smart electronic contact lenses.
\end{abstract}

() 2015 Elsevier B.V. All rights reserved.

\section{Introduction}

Enzymatic fuel cells (EFCs) have been envisioned as electrical power sources for self-contained biomedical electronic devices

\footnotetext{
* Corresponding author. Biomedical Sciences, Health and Society, Malmö University, 20560 Malmö, Sweden.

E-mail address: sergey.shleev@mah.se (S. Shleev).
}

[1-3]. EFCs have some great advantages due to the special properties of oxidoreductases - renewable biocatalysts, viz. their high specificity and selectivity, as well as specific activity [4]. First, EFCs can potentially be produced at low cost and have great possibilities for miniaturisation. Second, the products of EFC operation are usually less harmful to the body compared to the products of conventional FCs [5]. Finally, a direct electron transfer (DET) based approach can allow great simplification in the designed biodevice, excluding the need for membranes and toxic mediators [6]. 
Nanostructuring of electrode surfaces is very important in order to improve the basic characteristics of EFCs $[7,8]$. However, usually simple immobilisation of separately synthesised nanomaterials on electrode surfaces is performed. Because of its irreproducibility, this technology cannot be directly used on an industrial level. This is one of the main problems with biofuel cell technology that presently hinder real practical applications of EFCs. Here we show the fabrication and characterisation of nanostructured electrodes for EFCs using nanoimprint lithography (NIL), which ensures a wellcontrolled nanostructure geometry of the electrodes on an industrial level. NIL is a parallel patterning technique capable of making features as small as 2-3 nm or even less in a fast, reproducible, scalable, and economical way [9]. NIL allows easy surface structuring over large areas (up to 6 inches in diameter) with very high resolution (a few $\mathrm{nm}$ ) and industrial scale throughput. In our studies we used well-known anodic and cathodic redox enzymes, cellobiose dehydrogenase ( $\mathrm{CDH}$ ) and bilirubin oxidase (BOx), which are among the main biocatalysts exploited nowadays to design DET-based glucose/oxygen EFCs $[6,10]$.

For certain applications, e.g. smart contact lenses [11], EFC should be biocompatible, flexible, and transparent [10,12]. In spite of many reports in the literature concerning transparent $[13,14]$ and especially flexible biological FCs (BFCs) [15-21], these biodevices cannot be used directly to power electronic contact lenses for many reasons. First, previously designed transparent BFCs were built on rigid materials, such as titanium oxide coated [13] or indium oxide coated [14] conductive glasses. Second, most flexible EFCs were macroscale opaque biodevices [17,18,20,21]. Finally, to the best of our knowledge, all previous flexible biofuel cells were built using metal or carbon nanomaterials, viz. nanoparticles and nanotubes, immobilised on electrode surfaces. However, there is an ongoing debate concerning the biocompatibility and safety of these nanomaterials [22-24]. Below we detail safe, transparent, and flexible, glucose/oxygen EFCs with nanostructured electrodes that can be reproducibly fabricated with industrial scale throughput.

\section{Experimental}

Unless otherwise specified, all chemicals were purchased from Sigma-Aldrich GmbH (Schnelldorf, Germany). Acetone was purchased from Merck KGaA (Darmstadt, Germany). All solutions were prepared using water purified with a PURELAB UHQ II system from ELGA Labwater (High Wycombe, UK). Nitrogen was obtained from AGA Gas AB (Sundbyberg, Sweden). For electrochemical measurements nitrogen was additionally purified using Gas Clean Filters from Varian BV (Middelburg, The Netherlands). Corynascus thermophilus $\mathrm{CDH}$ and Myrothecium verrucaria BOx were employed as anodic and cathodic biocatalysts, respectively. Purified $\mathrm{CDH}$ and BOx were received as kind gifts from BOKU-University (Austria) and Amano Enzyme Inc. (Japan), respectively.

The intermediate polymer stamp (IPS ${ }^{\circledR}$ ) sheets were provided by Obducat Technologies AB (Lund, Sweden). According to the provider the polymer is biocompatible. The polymers sheets, roughly $20 \times 20 \mathrm{~cm}$, either bare or imprinted as described below, henceforth called planar and nanostructured sheets, respectively, cleaned in acetone at room temperature for $2 \mathrm{~min}$, and dried with $\mathrm{N}_{2}$ gas. The sheets were imprinted using nanoimprint lithography (NIL) at $160{ }^{\circ} \mathrm{C}$ and 50 bar for $120 \mathrm{~s}$ and subsequently demolded at $115^{\circ} \mathrm{C}$ for $40 \mathrm{~s}$. The imprint was performed using a $6^{\prime \prime}$-Imprinter from Obducat Technologies AB. The nickel nanoimprint stamp was purchased from NIL Technologies ApS (Kongens Lyngby, Denmark). The stamp had undergone an anti-stick treatment at Obducat Technologies $A B$, applying a thin self-assembling monolayer film of fluorinated alkyl phosphoric acid derivatives, as described in Ref. [25]. Then, both planar and nanostructured sheets were covered with $5 \mathrm{~nm}$ Ti followed by $20 \mathrm{~nm}$ of Au by a thermal evaporator in a custom built system at low pressure. Titanium (Ti) wire (99.99+\%) was purchased from Goodfellow Cambridge Ltd. (Huntingdon, England), while the gold ( $\mathrm{Au}$ ) nuggets (99.9999\%) were purchased from Dahlgren Ädelmetall AB (Malmö, Sweden). The metal films were evaporated at a base pressure of $<10^{-6} \mathrm{mbar}$ with an average deposition rate of 1 and $10 \AA \mathrm{s}^{-1}$ for Ti and $\mathrm{Au}$, respectively. The $5 \mathrm{~nm}$ thick layer of Ti was deposited to promote the adhesion of the Au layer on the substrates. For FC fabrication, however, two separate electrodes were built on a single polymer substrate by the addition of a plastic separator during Au evaporation (Fig. 1). Moreover, two Au current collectors $2 \mathrm{~mm}$ in width were also additionally fabricated by evaporating a $300 \mathrm{~nm}$ Au layer on the inside of two Au electrodes, while the rest of the surface was covered (protected) during this evaporation step (Fig. 1, two white arrows). A geometric area (also called a two dimensional projected area) of the electrodes was determined by direct precise geometric measurements using a vernier caliper from Mitutoyo Scandinavia AB (Upplands Väsby, Sweden).

Surface morphology was studied using scanning electron microscopy (SEM) and atomic force microscopy (AFM). SEM images were taken using a Nova NanoLab 600 Dual Beam focused ion beam and a scanning electron microscope (FIB/SEM) from FEI Company (Hillsboro, Oregon, USA). Images were taken with an immersion lens at an acceleration voltage of $30 \mathrm{kV}$ and a beam current of $2.4 \mathrm{nA}$. AFM images were obtained using a Multimode VIII SPM with a Nanoscope V control unit from Bruker AXS (Santa Barbara, CA, USA). The AFM was operated in the ScanAsyst mode. All images were obtained in air and at room temperature. Triangular silicon nitride cantilevers with a nominal spring constant of $0.4 \mathrm{~N} \mathrm{~m}^{-1}$ (ScanAsyst Air probes, Bruker AXS) were employed in all measurements. Analysis and processing of AFM images was performed with the WSxM software package [26]. Image processing consisted of plane subtraction, equalisation and 3D representation. Optical properties of the transparent electrodes were studied spectrophotometrically using a Helios $\beta$ spectrophotometer from Thermo Electron Corporation (Marietta, OH, USA) at room temperature.

Electrochemical measurements of bare Au electrodes were performed in $0.5 \mathrm{M} \mathrm{H}_{2} \mathrm{SO}_{4}$ to clean the Au electrodes and assure uniform Au surfaces on a molecular level, as well as to determine the real (also called microscopic or electrochemically active) electrode area [27]. For this purpose fabricated Au electrodes were connected as working electrodes to a $\mu$ Autolab Type III/FRA2 potentiostat/galvanostat from MetrohmAutolab B.V. (Utrecht, The Netherlands) using Au-plated alligator clips model 3289-2 from Pomona Electronics (Everett, WA, USA) attached to the corners of the electrodes (Fig. 1A). For the EFC investigation the biodevices were also connected using two clips attached to the current collectors (Fig. 1B). Separate Au electrodes, as well as FCs, were subjected to an oxidation-reduction cycle in $0.5 \mathrm{M} \mathrm{H}_{2} \mathrm{SO}_{4}$ between 0 and $+1.9 \mathrm{~V}$ at a scan rate of $0.1 \mathrm{~V} \mathrm{~s}^{-1}$ for 10 cycles (Fig. $2 \mathrm{~A}$ ) prior to biomodification. While using a three-electrode configuration, an $\mathrm{Hg}\left|\mathrm{Hg}_{2} \mathrm{Cl}_{2}\right| \mathrm{KCl}_{\text {sat }}$ (SCE, $242 \mathrm{mV}$ vs. normal hydrogen electrode (NHE)) and a platinum wire mesh were applied as reference and counter electrodes, respectively. However, all potentials in the present work are re-calculated and they are always given $v$ s. NHE. The real surface area of the electrodes was calculated from the experimentally measured charge associated with the Au oxide reduction process performed by running cyclic voltammetry (Fig. 2A). A cathodic current peak at $1.1 \mathrm{~V}$ related to the reduction of the Au oxide was integrated to calculate the practical charge density. The theoretical charge density associated with this process was taken to be $390 \mu \mathrm{C} \mathrm{cm}^{-2}$ [27].

Next the Au electrodes were modified with suitable biocatalysts. For cathode biomodification, a BOx solution $\left(1 \mathrm{mg} \mathrm{mL}^{-1}\right.$ in $10 \mathrm{mM}$ 

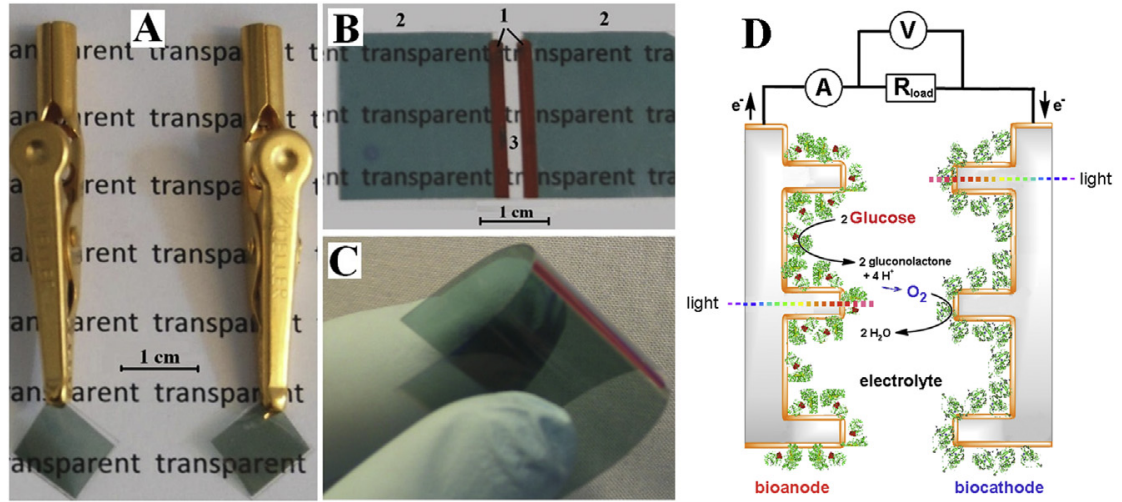

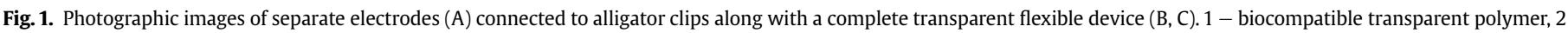

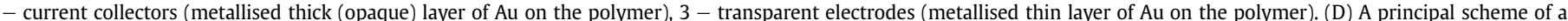
compartment- and mediator-less, flexible and transparent glucose/oxygen enzymatic fuel cell based on nanostructured electrodes.

sodium phosphate buffer, $\mathrm{pH}$ 6.0) was placed onto the electrode to fully cover its surface for $1 \mathrm{~h}$ at room temperature. Afterwards the electrodes were carefully rinsed with $50 \mathrm{mM}$ phosphate buffer, $\mathrm{pH}$ 7.4. For anode biomodification, a volumetric $1: 1$ mixture of $1 \mathrm{mM} \mathrm{4-}$ aminothiophenol ethanol and $1 \mathrm{mM}$ 4-mercaptobenzoic ethanol was placed onto the electrode surface for $1 \mathrm{~h}$ at room temperature. The resulting thiol-modified electrodes were thoroughly rinsed with ethanol and gently dried with a flux of nitrogen. Then, $1 \mu \mathrm{L}$ of glutaraldehyde and $2 \mu \mathrm{L}$ of $\mathrm{CDH}\left(8.4 \mathrm{mg} \mathrm{mL}^{-1}\right)$ were cast and carefully mixed well on the electrode. The cross-linking reaction of $\mathrm{CDH}$ was allowed to take place for $1 \mathrm{~h}$. Afterwards the electrodes were carefully rinsed with $50 \mathrm{mM}$ phosphate buffer, $\mathrm{pH}$ 7.4. It is also important to note that the electrodes were not allowed to dry at any moment after biomodification.

Electrochemical characterisation of bioanodes, biocathodes, and EFCs was performed using a a $\mu$ Autolab Type III/FRA2 potentiostat/ galvanostat by recording linear sweep voltammograms and chronoamperograms. Operational stability tests were performed by chronoamperometry using applied potentials of $0.24 \mathrm{~V}$ and $0.40 \mathrm{~V}$ for bioanodes and biocathodes, respectively. At these potentials the bioelectrodes produced close to maximal power outputs without the influence of non-bioelectrocatalytic processes (vide infra). To characterise the EFC performance, steady-state voltage measurements were performed with different calibrated resistors, 1-100 M $\Omega$, from Velleman Inc. (Forth Worth, TX, USA). The resistors were calibrated using the potentiostat/galvanostat, which has an input impedance $>100 \mathrm{G} \Omega$. The system was allowed 5 min to reach equilibrium for each point. The stability of the EFC was determined by monitoring the change in voltage when a constant load was applied to the EFC to obtain an operating voltage of $0.35 \mathrm{~V}$, i.e. the voltage corresponding to the maximal performance of the biodevice.

No less than 50 separate bioelectrodes and three complete EFCs (Fig. 1) in total were fabricated during our studies. To estimate statistical parameters, averaged OCP and OCV values of bioelectrodes and EFCs were based on at least three measurements, respectively. Typical linear sweep voltammograms were obtained by averaging over three different measurements (curves). However, single operational stability tests were carried out for all types of biodevices, i.e. a biocathode, a bioanode, and a complete EFC.

\section{Results and discussion}

In our previous studies, detailed investigations of nanostructured Au electrodes based on Au nanoparticles (AuNPs), CDH and BOx modified AuNP based electrodes, as well as glucose/oxygen AuNPs based EFCs were performed [28]. In the present study transparent and flexible bioelectrodes and biodevices were fabricated and evaluated following similar methodology (Fig. 1D). However, prior to that, the NIL electrodes were carefully analysed and NIL modified surfaces were compared to AuNP modified surfaces. As evidenced from Fig. 1, the devices were transparent (29-54\% transparency in the visible spectrum, Fig. 2B). On the one
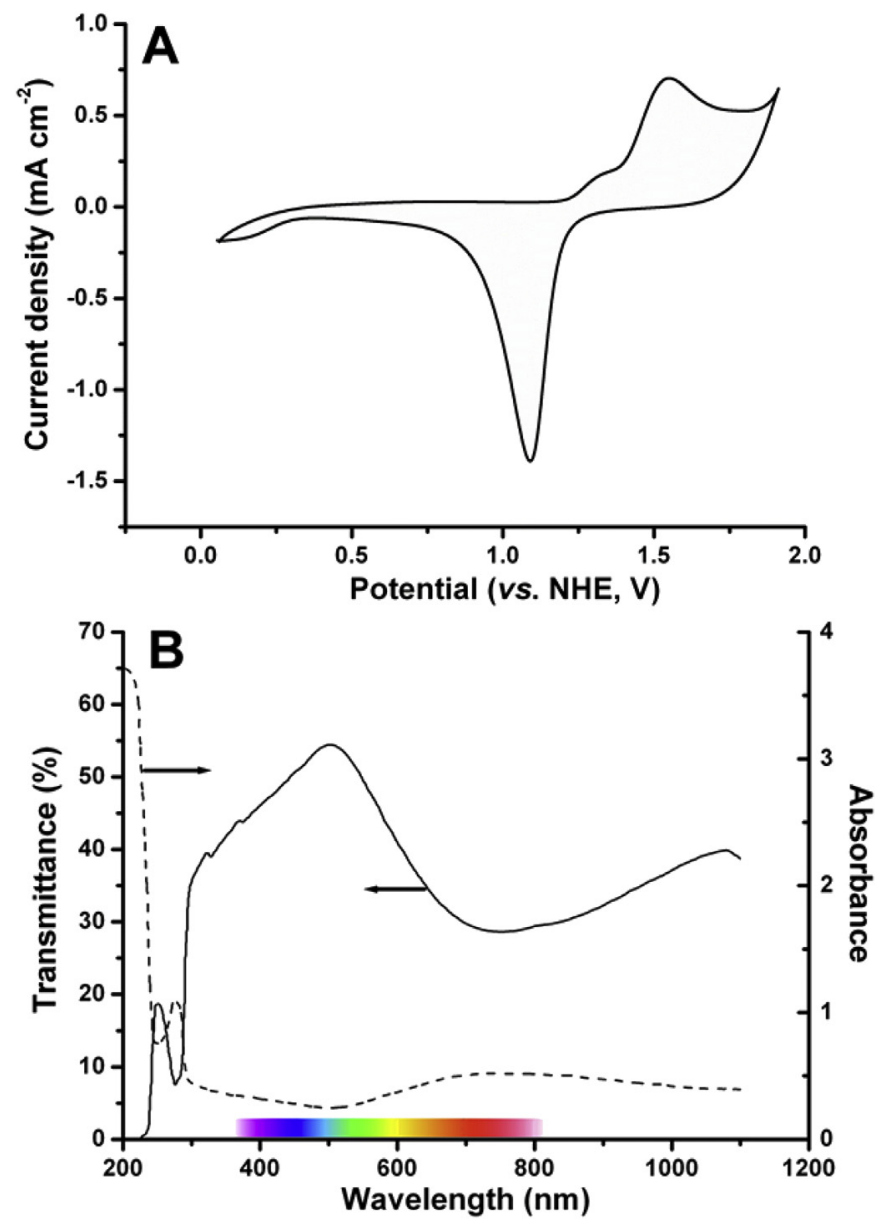

Fig. 2. (A) Typical cyclic voltammogram of Au electrodes submerged in $0.5 \mathrm{M} \mathrm{H}_{2} \mathrm{SO}_{4}$. (B) UV-VIS-NIR spectrum of Au electrodes. 
hand, while AuNP modified electrodes had a huge real surface area (at least 200 times higher compared to the geometric one [28]), the electrochemically active surface area of NIL modified electrodes was only 7-fold higher than the geometric area, as revealed by electrochemical measurements of Au electrodes in $\mathrm{H}_{2} \mathrm{SO}_{4}$ (Fig. 2A; vide supra). On the other hand, while AuNP modified electrodes had a very rough surface with uncontrolled nano-features of different sizes and diameters, NIL modification resulted in the formation of a regular, well-ordered $2 \mathrm{D}$ hexagonal lattice of nano-cavities. The centres of the nano-cavities were separated by an average distance of $c a .293 \mathrm{~nm}$, whereas their depth was $230 \pm 13 \mathrm{~nm}$ or $148 \pm 24 \mathrm{~nm}$ depending on the directions defined by the two different primitive translation vectors of the lattice, as revealed by AFM and SEM measurements ( $c f$. Fig. 3 presented herein with Figs. 2 and 3 in Ref. [28]).

Electrochemical investigations of bare Au electrodes were performed in air-saturated $50 \mathrm{mM}$ potassium phosphate buffer, $\mathrm{pH} 7.4$, containing $150 \mathrm{mM} \mathrm{NaCl}$ (PBS) along with $5 \mathrm{mM}$ glucose in the potential range of $0.05-0.85 \mathrm{~V} v$ s. NHE (Fig. 4). Well pronounced electrocatalytic oxidation of glucose was observed at potentials above $0.45 \mathrm{~V}$, whereas reduction of $\mathrm{O}_{2}$ was observed at potentials below $0.2 \mathrm{~V} v s$. NHE, which is in good agreement with the literature [29-31]. Moreover, quite significant anodic currents were registered at potentials above $0.8 \mathrm{~V}$ (Fig. 4B). One of the reasonable explanations for the current ramp near the positive potential could be the anodic stripping of $\mathrm{Au}$ in chloride anion containing buffer. However, since the Au layer is very thin, stripping should be visually observed. However, Au stripping was not observed, when cycling electrodes in the buffer in a broad potential range. Thus, more likely explanation is water electrooxidation on the electrode surface at high redox potentials. Indeed, these potential regions were excluded from further investigations of separate biomodified electrodes (Fig. 4). In $5 \mathrm{mM}$ glucose containing air-saturated PBS, $\mathrm{pH} 7.4$, the open-circuit potentials (OCPs) of bioanodes and biocathodes were found to be $0.11 \pm 0.08 \mathrm{~V}$ and $0.74 \mathrm{~V} \pm 0.05 \mathrm{~V} v s$. NHE, respectively. Thus, one can estimate the theoretical OCV of the EFC to be about $0.63 \mathrm{~V}$. This value is almost twice as low as the maximal thermodynamically possible voltage based on standard redox potentials of gluconolactone/glucose and oxygen/water couples ( $0.05 \mathrm{~V}$ and $1.23 \mathrm{~V}$ vs. NHE, respectively). Whereas OCP values of biocathodes were quite close to the redox equilibrium potential of the catalysed half-reaction under experimental conditions (0.79 $\mathrm{V}$ at $\left.\mathrm{pH} 7.4,25{ }^{\circ} \mathrm{C}[32]\right)$, a huge loss was registered on the bioanode values. This is explained by the redox potential of the anodic biocatalyst, $\mathrm{CDH}$, being equal to $0.15 \mathrm{~V}$ [33], which is $0.54 \mathrm{~V}$ higher than the redox equilibrium potential of the catalysed half-
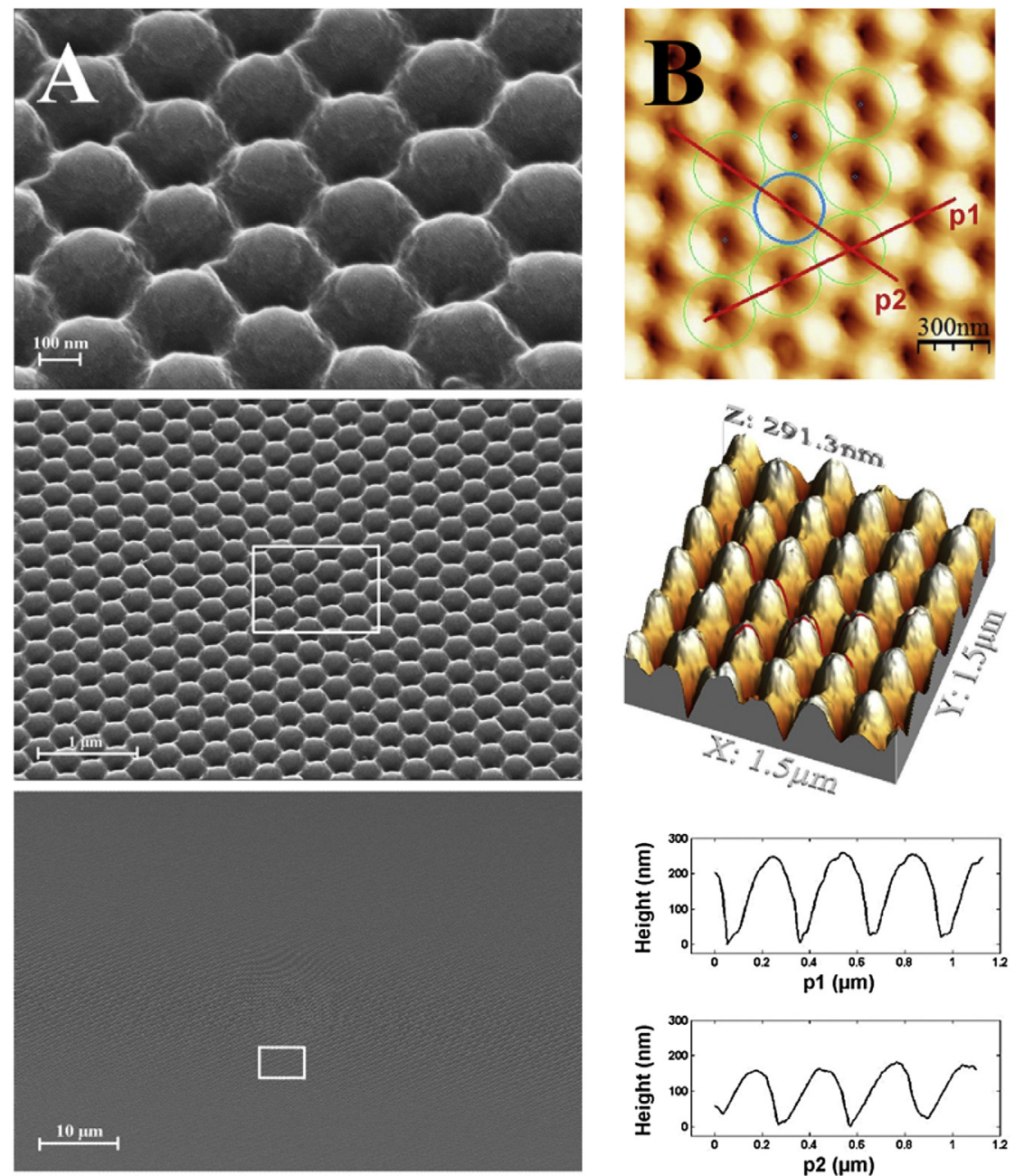

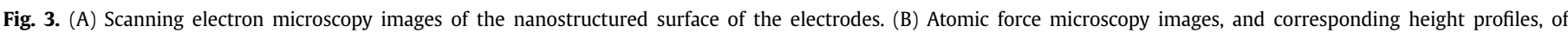

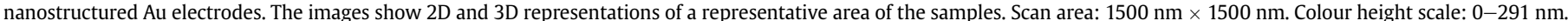

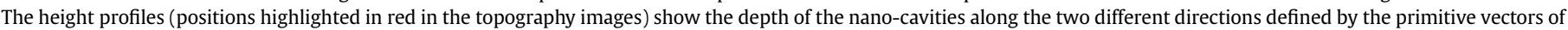

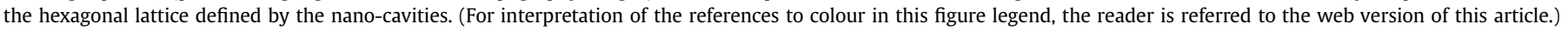



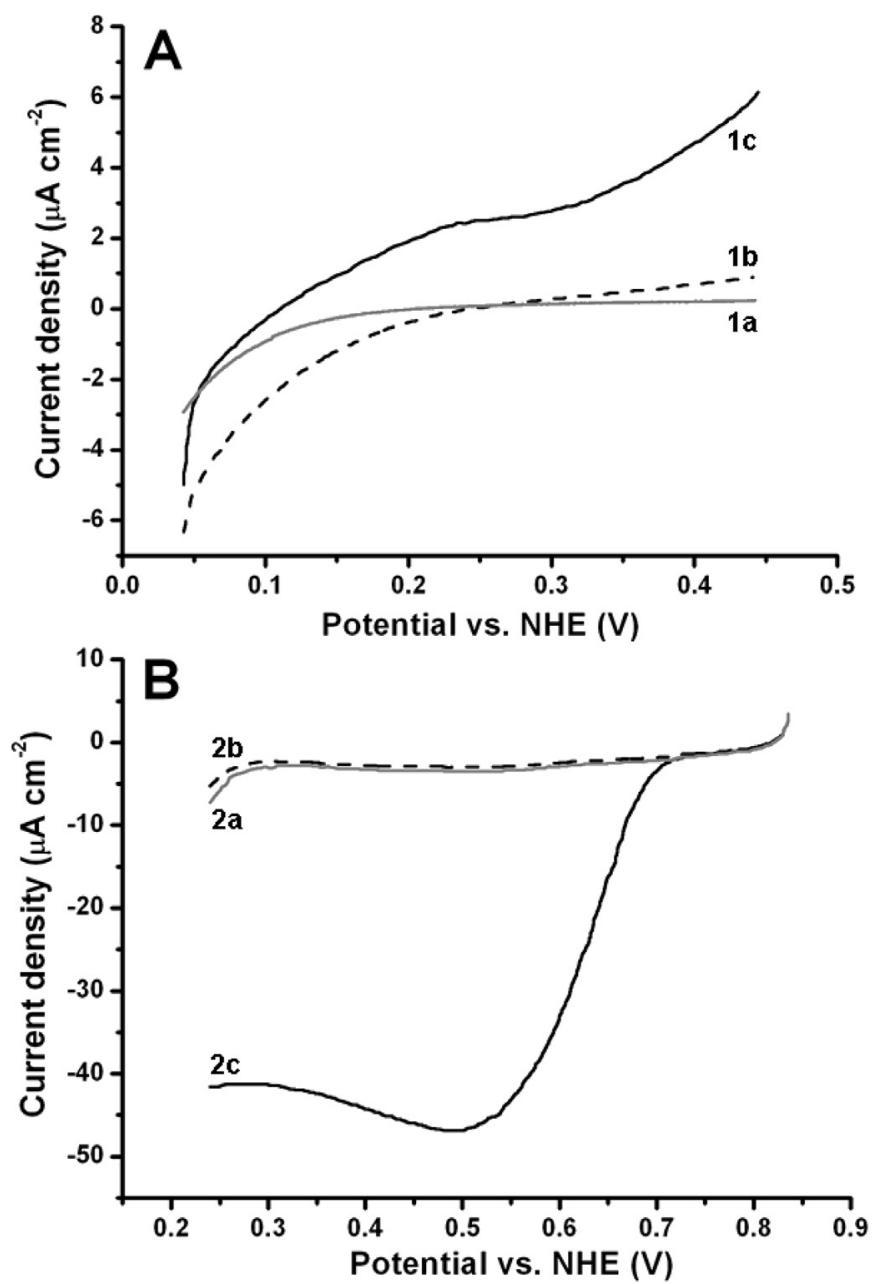

Fig. 4. (A) Linear sweep voltammograms of anodes (grey curve 1a; without adsorbed $\mathrm{CDH}$ ) and bioanodes (solid curve 1c and dashed curve $1 \mathrm{~b}$; biomodified electrodes) operating in PBS with (solid curve 1c and grey curve 1a) and without (dashed curve 1b) $5 \mathrm{mM}$ glucose. (B) Linear sweep voltammograms of cathodes (grey curve 2a; without adsorbed BOx) and biocathodes (solid curve 2c and dashed curve $2 \mathrm{~b}$; biomodified electrodes) operating in PBS with (solid curve $2 \mathrm{c}$ and grey curve $2 \mathrm{a}$ ) and without (dashed curve 2b) $0.25 \mathrm{mM}$ oxygen.

reaction under experimental conditions $\left(-0.39 \mathrm{~V}\right.$ at $\mathrm{pH} 7.4,25^{\circ} \mathrm{C}$ [4]). Well-pronounced bioelectrocatalytic currents were registered when CVs were recorded in the potential range of $0.05 \mathrm{~V}-0.45 \mathrm{~V}$ and $0.35 \mathrm{~V}-0.85 \mathrm{~V}$ for bioanodes and biocathodes, respectively (Fig. 4), with maximal registered bioelectrocatalytic current densities of $3 \mu \mathrm{A} \mathrm{cm}^{-2}$ and $44 \mu \mathrm{A} \mathrm{cm}^{-2}$ at $0.3 \mathrm{~V}$ and $0.5 \mathrm{~V}$ (all current densities in the text and figures are given using the geometric (projected) surface area of electrodes). When glucose-free buffer was used and oxygen was removed by saturating the PBS with nitrogen instead of air, the electrocatalytic currents vanished, confirming their biocatalytic origins.

It should be emphasised that while OCP values for both imprinted and un-imprinted biomodified electrodes were almost identical, three times as lower current densities were registered, when planar (un-imprinted) electrodes modified with redox enzymes were used, viz. $1 \mu \mathrm{A} \mathrm{cm} \mathrm{cm}^{-2}$ and $15 \mu \mathrm{A} \mathrm{cm} \mathrm{cm}^{-2}$ at $0.3 \mathrm{~V}$ and $0.5 \mathrm{~V}$ for bioanode and biocathode, respectively. Thus, electrode nanostructuring is a crucial step to obtain acceptable current densities, especially in the case of CDH based bioanodes. However, it is also interesting to note that nanostructuring enhances current outputs due to increase in real surface area. If one compares current densities of un-imprinted and imprinted biomodified electrodes and their roughness factors ( 2 and 7 , respectively), clear correlation can be observed.

The operational stability of the bioanodes was much better compared to the biocathodes, which lost $50 \%$ of their bioelectrocatalytic output in $14 \mathrm{~h}$ ( $c f$. curves 1 and 2 in Fig. 5A). Following our recent report, one could suggest that the poor stability of the biocathodes was, in all likelihood, attributable to BOx deactivation on the bare Au surface [34], whereas CDH was protected from the metal surface by the thiol layer.

The registered characteristics of transparent and flexible EFCs operating in $5 \mathrm{mM}$ glucose and $150 \mathrm{mM}$ chloride containing neutral buffer, viz. $0.63 \mathrm{~V}$ OCV value, $0.6 \mu \mathrm{W} \mathrm{cm} \mathrm{cm}^{-2}$ maximal power density at a cell voltage of $0.35 \mathrm{~V}$ (Fig. $5 \mathrm{~B}$ ), were close to the main parameters predicted based on detailed experimental studies of separate bioanodes and biocathodes (vide supra). The stability test revealed that EFCs could still deliver at least $0.5 \mu \mathrm{W} \mathrm{cm}{ }^{-2}$ after $12 \mathrm{~h}$ of continuous operation, following, however, the stability trend of the bioanode ( $c f$. curves 1 and 3 in Fig. 5A). Even if the biocathode was shown to be the less stable electrode, this is not surprising because both bioelectrodes had equal geometric areas (Fig. 1), whereas the bioelectrocatalytic current densities of the bioanodes were 15 -fold lower compared to the biocathodes.

Even though the obtained power output of transparent and
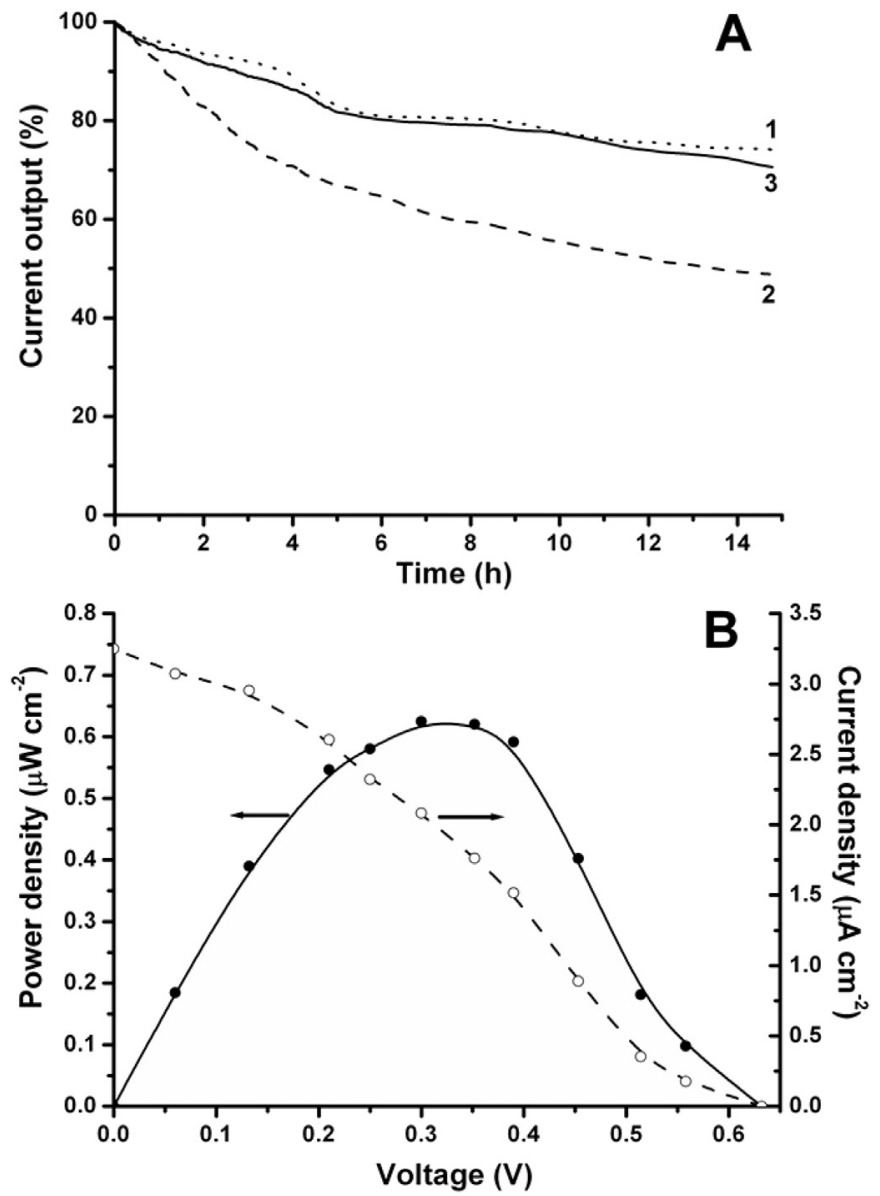

Fig. 5. (A) Operational stability of a bioanode (dotted curve 1), a biocathode (dashed curve 2), and an EFC (solid curve 3) operating in air saturated PBS containing $5 \mathrm{mM}$ glucose. (B) Typical response for glucose/oxygen EFC operating in air saturated PBS containing $5 \mathrm{mM}$ glucose, showing the current output (right axis, dashed curve, empty circles) and power output (left axis, solid curve, filled circles) as a function of the voltage. 
flexible EFC is low, given the fast paced development of modern electronics, such as a wireless autonomous system with an average power consumption of only $5.3 \mathrm{nW}$ being reported [35], an EFC as described herein can still be practically useful. Moreover, transparent and flexible glucose/oxygen EFC could also potentially be employed as a sensing unit by itself, e.g. as a self-powered biosensor $[14,36]$. Because the bioanode limits the power output of the biodevice, its power is directly connected to glucose concentration. In one possible design of a glucose sensing electronic contact lens, such EFC might be used as a glucose biosensor, whereas electric power for data transmission would be wirelessly delivered overthe-air using an external power source and an antenna incorporated into a lens $[12,37]$. Furthermore, the observed operational stability is far from perfect, but fully enough for disposable one-day usage contact lenses. The limited long-term stability of EFCs is a serious problem, caused by the inherent short active lifetimes of enzymes [4]. The employment of EFCs in non-invasive (ex vivo) contact situations is a promising biomedical application where most of the in vivo shortcomings, such as insufficient operational lifetime, immunoresponse, encapsulation, etc., are non-issues $[6,10]$. It is also important to emphasise that glucose is not used as an energy source by cornea cells [38]. Thus, there is a barrier to reduce the glucose concentration in tears compared to blood in order, for instance, to prevent possible bacterial infection of the eye. Thus, glucose utilisation by EFCs incorporated into contact lenses might have a positive effect on eye health.

In addition to the positive aspects described just above, some disadvantages of fabricated EFCs for direct application in human contact lenses should also be mentioned. One of the main drawbacks is the submerged biocathode, which uses molecular oxygen from the solution. For human eyes the oxygen concentration in lachrymal liquid (tears) is crucial [38]. Indeed, modern contact lenses are oxygen permeable [39]. To overcome this serious problem, instead of submerged biodevices, air-breathing biocathodes could be used [40-43]. Another drawback is the low performance of transparent and flexible EFCs described herein. However, in our previous studies we have shown that the average electric power extractable from human tears (calculations were done using tear flow rate and glucose concentration ranges) is just on the order of a few $\mu \mathrm{W}$ [11]. Thus, even low performing FCs would be able to convert all the chemical energy available in tears in the form of glucose into electric energy.

\section{Conclusions}

We present experimental proofs that flexible, transparent, nanostructured, membrane-less and mediator-free glucose/oxygen enzymatic fuel cells, which can be reproducibly fabricated with industrial scale throughput, do generate electrical power from glucose and molecular oxygen as biofuel and biooxidant, respectively. This type of biodevice could potentially be used in smart electronic contact lenses, which could provide a great benefit for continuous biomedical monitoring. Work is currently ongoing in our laboratory to increase the performance of the EFC and, as the next step, to design biodevices using real human contact lenses with nanostructured bioanodes based on GDH and air breathing biocathodes based on BOx.

\section{Acknowledgements}

The authors thank Dr. Roland Ludwig and Amano Enzyme Inc. for $\mathrm{CDH}$ and the Amano 3 preparation of BOx, respectively. This work was supported financially by the Russian Foundation for Basic Research (14-04-32235 to DP and 13-04-12063 to DP, AL, and SS), by the European Commission (PEOPLE-2013-ITN-607793) to EG-A and SS, by the Swedish Research Council (2013-6006) to SS, and by the Nanometer Structure Consortium at Lund University (nmC@LU) to IM, MG, DBS, and LM.

\section{References}

[1] S.C. Barton, J. Gallaway, P. Atanassov, Chem. Rev. 104 (2004) 4867-4886.

[2] A. Heller, Phys. Chem. Chem. Phys. 6 (2004) 209-216.

[3] M. Falk, C.W. Narvaez Villarrubia, S. Babanova, P. Atanassov, S. Shleev, ChemPhysChem 14 (2013) 2045-2058.

[4] R.H. Garrett, C.M. Grisham, Biochemistry, Brooks Cole, Boston, 2012.

[5] M. Falk, S. Shleev, C.W. Narvaez Villarrubia, S. Babanova, P. Atanassov, Biological fuel cells for biomedical applications: colonizing the animal kingdom, in: H. Luckarift, P. Atanassov, G. Johnson (Eds.), Enzymatic Fuel Cells, John Wiley \& Sons, Hoboken, New Jersey, 2014, pp. 422-450.

[6] M. Falk, Z. Blum, S. Shleev, Electrochim. Acta 82 (2012) 191-202.

[7] S.D. Minteer, Top. Catal. 55 (2012) 1157-1161.

[8] A. de Poulpiquet, A. Ciaccafava, E. Lojou, Electrochim. Acta 126 (2014) $104-114$.

[9] H. Schift, J. Vac. Sci. Technol. B 26 (2008) 458-480.

[10] M. Falk, D. Pankratov, Z. Blum, S. Shleev, Direct-electron-transfer-based emzymatic fuel cells in vitro, ex vivo, and in vivo, in: E. Katz (Ed.), Implantable Bioelectronics, Wiley-VCH, Weinheim, 2014, pp. 315-346.

[11] M. Falk, V. Andoralov, Z. Blum, J. Sotres, D.B. Suyatin, T. Ruzgas, T. Arnebrant S. Shleev, Biosens. Bioelectron. 37 (2012) 38-45.

[12] Z. Blum, D. Pankratov, S. Shleev, Expert Rev. Ophthalmol. 9 (2014) 269-273.

[13] Y. Amao, Y. Takeuchi, Int. J. Hydrogen Energy 33 (2008) 2845-2849.

[14] A. Zloczewska, A. Celebanska, K. Szot, D. Tomaszewska, M. Opallo, M. JonssonNiedziolka, Biosens. Bioelectron. 54 (2014) 455-461.

[15] T. Miyake, S. Yoshino, T. Yamada, K. Hata, M. Nishizawa, J. Am. Chem. Soc. 133 (2011) 5129-5134.

[16] T. Ruzgas, S. Shleev, T. Arnebrant, Flexible Biofuel Cell, Device and Method PCT Int. Appl. WO 2011-EP54554, 2011, p. 31.

[17] K. Haneda, S. Yoshino, T. Ofuji, T. Miyake, M. Nishizawa, Electrochim. Acta 82 (2012) 175-178.

[18] G.M. Olyveira, J.H. Kim, M.V.A. Martins, R.M. Iost, K.N. Chaudhari, J.-S. Yu, F.N. Crespilho, J. Nanosci. Nanotechnol. 12 (2012) 356-360.

[19] F.C.P.F. Sales, R.M. Iost, M.V.A. Martins, M.C. Almeida, F.N. Crespilho, Lab. Chip 13 (2013) 468-474

[20] I. Shitanda, S. Kato, Y. Hoshi, M. Itagaki, S. Tsujimura, Chem. Commun. 49 (2013) 11110-11112.

[21] X.E. Wu, Y.Z. Guo, M.Y. Chen, X.D. Chen, Electrochim. Acta 98 (2013) 20-24.

[22] V. Castranova, P.A. Schulte, R.D. Zumwalde, Acc. Chem. Res. 46 (2013) 642-649.

[23] F. Balas, RSC Green. Chem. Ser. 19 (2013) 213-222.

[24] Y. Song, S. Tang, Sci. World J. 11 (2011) 1821-1828.

[25] M. Keil, M. Beck, G. Frennesson, E. Theander, E. Bolmsjo, L. Montelius B. Heidari, J. Vac. Sci. Technol. B 22 (2004) 3283-3287.

[26] I. Horcas, R. Fernandez, J.M. Gomez-Rodriguez, J. Colchero, J. Gomez-Herrero, A.M. Baro, Rev. Sci. Instrum. 78 (2007), 013705/013701-013705/013708.

[27] S. Trasatti, O.A. Petrii, Pure Appl. Chem. 63 (1991) 711-734.

[28] V. Andoralov, M. Falk, B. Suyatin Dmitry, M. Granmo, J. Sotres, R. Ludwig, O. Popov Vladimir, J. Schouenborg, Z. Blum, S. Shleev, Sci. Rep. 3 (2013) 3270.

[29] A. Damjanovic, M.A. Genshaw, J.O.M. Bockris, J. Electroanal. Chem. Interfacial Electrochem. 15 (1967) 173-180.

[30] M.A. Genshaw, A. Damjanovic, J.O.M. Bockris, J. Electroanal. Chem. Interfacia Electrochem. 15 (1967) 163-172.

[31] T. Vidakovic-Koch, I. Ivanov, M. Falk, S. Shleev, T. Ruzgas, K. Sundmacher, Electroanalysis 23 (2011) 927-930.

[32] N. Watanabe, M.A.V. Devanathan, J. Electrochem. Soc. 111 (1964) 615-619.

[33] V. Coman, R. Ludwig, W. Harreither, D. Haltrich, L. Gorton, T. Ruzgas, S. Shleev, Fuel Cells 10 (2010) 9-16.

[34] D. Pankratov, J. Sotres, A. Barrantes, T. Arnebrant, S. Shleev, Langmuir 30 (2014) 2943-2951.

[35] G. Chen, H. Ghaed, R.-u. Haque, M. Wieckowski, Y. Kim, G. Kim, D. Fick, D. Kim, M. Seok, K. Wise, D. Blaauw, D. Sylvester, S.B.D. 17.6, in: IEEE International Solid-state Circuits Conference, 2011.

[36] E. Katz, A.F. Bueckmann, I. Willner, J. Am. Chem. Soc. 123 (2001) $10752-10753$.

[37] A.R. Lingley, M. Ali, Y. Liao, R. Mirjalili, M. Klonner, M. Sopanen, S. Suihkonen, T. Shen, B.P. Otis, H. Lipsanen, A. Parviz, J. Micromech. Microeng. 21 (2011), 125014/125011-125014/125018.

[38] E.R. Berman, Biochemistry of the Eye, Plenum Press, New York and London, 1991.

[39] A.J. Phillips, L. Speedwell, Contact Lenses, Elsevier, NewYork, 2007.

[40] R. Kontani, S. Tsujimura, K. Kano, Bioelectrochemistry 76 (2009) 10-13.

[41] S. Shleev, G. Shumakovich, O. Morozova, A. Yaropolov, Fuel Cells 10 (2010) 726-733.

[42] W. Gellett, J. Schumacher, M. Kesmez, D. Le, S.D. Minteer, J. Electrochem. Soc 157 (2010) B557-B562.

[43] G.P.M.K. Ciniciato, C. Lau, A. Cochrane, S.S. Sibbett, E.R. Gonzalez, P. Atanassov Electrochim. Acta 82 (2012) 208-213. 